\title{
Saúde emocional dos estudantes do ensino médio em distanciamento social decorrente da pandemia por COVID-19
}

\author{
Emotional health of high school students in social distance due to the COVID-19 pandemic
}

Salud emocional de estudiantes de secundaria en distancia social por la pandemia COVID-19

\author{
Aline Dias Gomes ${ }^{1 *}$, Claudia Mara de Melo Tavares²
}

Como citar esse artigo. Gomes, $\mathrm{AD}$; Tavares, CMM. Saúde emocional dos estudantes do ensino médio em distanciamento social decorrente da pandemia por COVID-19. Revista PróUniverSUS. 2020 Jul./Dez.; 11 (2): 192194.

\begin{abstract}
Resumo
Objetivos: Averiguar a percepção dos estudantes de ensino médio acerca das suas necessidades emocionais em face da pandemia por COVID 19; propor uma ação educativa que integre serviço de saúde-escola para promoção da saúde emocional dos adolescentes/escolares. Método: Estudo descritivo-exploratório com estudantes do ensino médio de uma escola pública da região metropolitana do Rio de Janeiro. Resultados esperados: Acredita-se que esse estudo contribua para a aproximação ou criação de redes de apoio para auxiliar os adolescentes, caso haja necessidade de apoio emocional. Produto: Esta pesquisa visa propor uma estratégia de aproximação entre estudantes, escola e atenção básica de saúde para apoio as necessidades emocionais dos adolescentes.
\end{abstract}

Palavras-chave: Saúde Mental, Adolescente, COVID-19.

\begin{abstract}
Objectives: To investigate the perception of high school students about their emotional needs in the face of the pandemic by COVID 19; propose an educational action that integrates health-school services to promote the emotional health of adolescents/ schoolchildren. Method: Descriptive-exploratory study with high school students from a public school in the metropolitan region of Rio de Janeiro. Expected results: It is believed that this study contributes to the approximation or creation of support networks to help adolescents, in case there is a need for emotional support. Product: This research aims to propose a strategy of approximation between students, school and primary health care to support the emotional needs of adolescents.

Keywords: Mental Health, Adolescent, COVID-19.
\end{abstract}

\section{Resumen}

Objetivos: Investigar la percepción de los estudiantes de secundaria sobre sus necesidades emocionales frente a la pandemia por COVID 19; proponer una acción educativa que integre los servicios de salud-escuela para promover la salud emocional de los adolescentes/escolares. Método: Estudio descriptivo-exploratorio con estudiantes de secundaria de una escuela pública de la región metropolitana de Río de Janeiro. Resultados esperados: Se cree que este estudio contribuye a la aproximación o creación de redes de apoyo para ayudar a los adolescentes, en caso de que exista necesidad de apoyo emocional. Producto: Esta investigación tiene como objetivo proponer una estrategia de aproximación entre estudiantes, escuela y atención primaria de salud para apoyar las necesidades emocionales de los adolescentes.

Palabras clave: Salud Mental, Adolescente, COVID-19.

${ }^{2}$ Enfermeira. Professora Titular do Departamento de Enfermagem Materno-Infantil e Psiquiatria da Escola de Enfermagem da Universidade Federal Fluminense. Programa de Mestrado Profissional em Ensino na Saúde/MPES, UFF, RJ, Brasil. Email: claudiamarauff@gmail.com ORCID: https://orcid.org/0000-0002-8416-6272 


\section{Introdução}

ASARSCoV-2 (COVID-19) surgiu no final do ano de 2019 em Whuan, China e espalhou-se rapidamente tornado-se uma pandemia que assola o mundo ao longo do ano de 2020.

Uma doença que acomete toda população, indiscriminadamente, apresentando desde uma forma assintomática até quadros graves de dificuldade respiratória em que o suporte ventilatório se faz necessário ${ }^{1}$.

AOrganização Mundial de Saúde (OMS) declarou como pandemia a doença causada pela COVID-19 em março de 2019. Atitudes como a quarentena e o isolamento social passaram a ser adotadas pelos governantes dos países afetados para tentar conter o avanço da doença ${ }^{2}$.

Sendo assim, ocorreu o fechamento de escolas, de universidades, de grande parte do comércio e toda população viu-se obrigada a adotar novos hábitos de vida como utilizar máscara e álcool gel, aprender a lavar corretamente as mãos, não sair de casa, não se reunir em grupos, evitar aglomerações ou lugares cheios, se sair de casa manter uma distância mínima de 2 metros entre as pessoas ${ }^{3}$.

A pandemia poderá deixar seqüelas tais como, transtornos de ansiedade, estresse, depressão e estas poderão ser maiores que o número de mortes, pois o distanciamento social prolongado impacta consideravelmente toda a população principalmente os grupos vulneráveis, como os adolescentes ${ }^{4-5}$.

As aulas presenciais foram substituídas por aulas em meios digitais após a publicação feita pelo Ministério da Educação da Portaria $\mathrm{n}^{0}$ 343, de 17 de março de $2020^{6}$. A suspensão das aulas presenciais restringiu um importante espaço de convivência dos adolescentes, a escola. Mas este vínculo ainda é mantido através das aulas remotas.

\section{Objetivos}

Averiguar a percepção dos estudantes de ensino médio acerca das suas necessidades emocionais em face da pandemia por COVID 19.

Propor uma ação educativa que integre serviço de saúde-escola para promoção da saúde emocional dos adolescentes / escolares.

\section{Metodologia}

Trata-se de uma pesquisa descritivo-exploratória sobre a promoção da saúde mental dos adolescentes que se encontram em distanciamento social e afastamento da escola em decorrência da pandemia por COVID-19.

Os participantes da pesquisa serão os adolescentes, alunos matriculados no ensino médio de uma escola pública da região metropolitana do Rio de Janeiro. A escola tem 362 alunos matriculados, 286 no turno da manhã e 76 alunos no turno da noite.

Critérios para inclusão dos participantes: estudantes do ensino médio, na fase compreendida como adolescência, que aceitem participar da pesquisa assinando o termo de consentimento livre e esclarecido.

$\mathrm{Na}$ impossibilidade da obtenção de dados por meio de entrevista presencial em função da pandemia, será elaborado e enviado para a direção da escola um instrumento de coleta de dados para análise em reunião de colegiado. Após a autorização da escola, da aquiescência dos pais e aprovação do Comitê de Ética em Pesquisa, o instrumento de coleta de dados via Google Forms, será inserido na Plataforma online do colégio.

Após a coleta, os dados serão analisados e categorizados, com base na técnica de análise de conteúdo de Bardin ${ }^{7}$ e categorizados de acordo com as temáticas.

A proposta de pesquisa será desenvolvida junto ao Programa de Pós-graduação do Mestrado Profissional em Ensino na Saúde Formação Docente Interdisciplinar para o SUS (MPES), da Escola de Enfermagem Aurora de Afonso Costa (EEAAC) - Universidade Federal Fluminense (UFF), linha de Pesquisa: Formação pedagógica em Saúde. O projeto de pesquisa foi aprovado em 30/10/2019 pelo Comitê de Ética e Pesquisa da Universidade Federal Fluminense, CAAE $\mathrm{n}^{\mathrm{o}}$ 17557719.3.0000.5243, parecer número 3.671.594.

\section{Resultados Esperados}

Acredita-se que o presente estudo contribua para a aproximação ou criação de redes de apoio aos adolescentes, ampliando a interação e colaboração entre a Estratégia Saúde da Família e a Escola.

Como produto deste estudo propõe-se uma roda de conversa virtual como espaço de esclarecimento e apoio para os alunos nos valendo de recursos artísticos para manejo das necessidades emocionais dos adolescentes frente ao isolamento social.

\section{Referências}

1. OMS. Organização Mundial da Saúde e OPAS - Organização PanAmericana de Saúde. Folha informativa COVID-19 - 2020: Doença causada pelo novo coronavírus. Acesso em 26 de setembro de 2020

2. Brasil. Ministério da Saúde (site). Painel Coronavírus. Ministério da Saúde, 2020. Disponível em: https://covid.saude.gov.br/. Acesso em 26 de setembro de 2020.

3. Faro A, Bahiano MA, Nakano TC, Reis C, Silva BFP, Vitti LS. COVID-19 e saúde mental: a emergência do cuidado. Estudos de Psicologia (Campinas). 2020; 37, e200074. Epub June 01, 2020. https://doi. 
org/10.1590/1982-0275202037e200074

4. Brooks SK, Webster RK, Smith LE, Woodland L, Wessely S, Greenberg N, Rubin, GJ. The psychological impact of quarantine and how to reduce it: rapid review of the evidence. Lancet Infect Dis. 2020; 395(10227): 912-920. doi: https://doi.org/10.1016/S0140-6736(20)30460-8

5. OMS. Organização Mundial da Saúde e OPAS - Organização PanAmericana de Saúde. Saúde Mental dos Adolescentes. Disponível em: https://www.paho.org/pt/topicos/saude-mental-dos-adolescentes. Acesso em 08 de novembro de 2020.

6. Brasil. Ministério da Educação. PORTARIA $N^{\circ} 343$, de 17 de março de 2020. Dispõe sobre a substituição das aulas presenciais por aulas em meios digitais enquanto durar a situação de pandemia do Novo Coronavírus - COVID-19. Disponível em: https://www.in.gov.br/en/web/dou/-/portarian-343-de-17-de-marco-de-2020-248564376. Acesso em 15 de novembro de 2020 .

7. Bardin L. Analise de conteúdo. São Paulo: Edições 70, 2011. 